CELL BIOLOGY

\section{Just the two of us}

An estimated $20-30 \%$ of proteins in the human genome are membrane proteins, and many of them need to interact with other proteins to elicit their biological effect (or effects), for example, by initiating a signaling cascade in response to an extracellular stimulus. Identifying the proteins that are necessary and sufficient for the biological effect to manifest can be very challenging. Petschnigg et al. have modified the splitubiquitin two-hybrid assay so that it can be used to detect interactions between a membrane protein and other proteins in mammalian cells. The authors showed that their method could be used to confirm that a GPCR- $\beta$-arrestin interaction only occurs in the presence of a small-molecule agonist and that a growth factor-adaptor protein interaction only takes place when the growth factor is phosphorylated at the proper position. They then determined that the assay could be scaled up; by screening 205 proteins that were computationally predicted to interact with EGFR, they identified 124 putative EGFR binders, many of which were previously known to bind EGFR or subsequently shown, in follow-up experiments, to interact with this growth factor. The authors believe that their method could be used to help unravel large-scale proteinprotein interaction networks and facilitate the development of drugs for diseases that are caused by the mutation or dysregulation of membrane proteins.

\section{APOPTOSIS}

\section{A channel off-target}

Nature doi:10.1038/nature13147

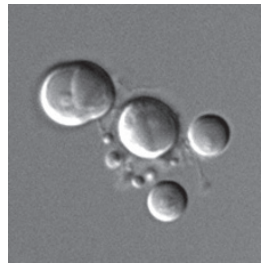

PANX1 is a member of the newly identified family of plasma membrane channels that allows the passage of small molecules such as nucleotides, which are implicated in processes such as neutrophil activation. During apoptosis, nucleotides passing through PANX1 to the extracellular space act as signals to recruit phagocytes to complete the cell death program. Poon et al. set out to look for drug-like compounds that target PANX1 as these could prove useful for a range of diseases linked to these processes. Using a flow cytometry-based screen, the authors identified three potent inhibitors of PANX1 transport function. One of them was the quinolonebased antibiotic trovafloxacin, known to target bacterial topoisomerases. Several experiments, including patch clamping, indicated that trovafloxacin directly inhibits PANX1 and is specific for this channel. Thus, PANX1 may be the mammalian target associated with the serious side effects known to be caused by trovafloxacin use as an antibiotic. The authors found two additional quinolone antibiotics, difloxacin and tosufloxacin, with PANX1targeting mechanisms similar to that of trovafloxacin. Using trovafloxacin, the authors next found a role for PANX1 in the regulation of the poorly characterized apoptotic cell disassembly into so-called apoptotic bodies and also defined an unappreciated early step of the apoptotic pathway involving PANX1mediated downregulation of long string-like membrane blebs that they called 'apoptopodia'. These results help define a liability with quinolone antibiotics as well as an early step of apoptosis in which PANX1 acts in ordered disassembly.

\section{NATURAL PRODUCTS}

\section{A painful discovery}

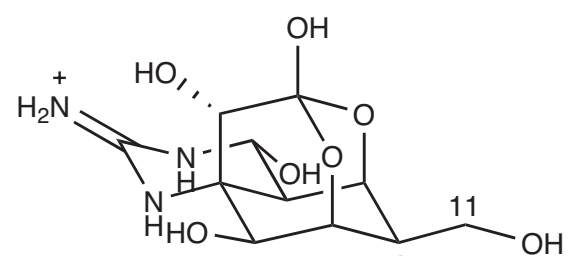

Marine animals use a diversity of potent neurotoxins to disable their prey. One such neurotoxin, tetrodotoxin, acts by blocking voltage-gated sodium channels with high affinity and specificity. As these channels are associated with chronic pain, tetrodotoxin and its analogs show promise as potential analgesics. However, the molecular determinants of activity, such as whether all six hydroxyl groups contribute to function, remain unclear. In a search for more information, Kudo et al. now report the discovery of 6-deoxytetrodotoxin, which lacks a hydroxyl group at the $\mathrm{C} 6$ position, from pufferfish. A unique HPLC retention time as compared to other deoxy analogs along with NMR data allowed
J. Nat. Prod. doi:10.1021/np401097n the authors to assign the compound's structure, which is a dynamic equilibrium of the hemilactal and lactone conformations. Profiling of five animal samples indicated that the new analog was present at similar concentrations as other analogs, with particularly high concentrations found in the posterior salivary gland of the Australian blue-ringed octopus. Activity assays indicated that 6-deoxytetrodotoxin was only threefold less effective than the parent compound, whereas the loss of the neighboring $\mathrm{C} 11$ hydroxyl group (from either 6-deoxytetrodotoxin or tetrodotoxin) leads to a $\sim 60$-fold drop in activity, indicating that the C6 group is not critical for function. This study provides the first direct test of the C6 modification and raises new questions about the biosynthetic pathway to this highly decorated molecule.

\section{KINASE REGULATION}

\section{Switching GSK3 off}

eLIFE doi:10.7554/eLife.01998

Glycogen synthase kinase-3 (GSK3) is a constitutively active kinase that regulates diverse processes such as glucose homeostasis and development. Activation of insulin or Wnt $/ \beta$-catenin signaling results in the inhibition of GSK3 activity through distinct mechanisms: insulin signaling turns on Akt expression, which phosphorylates GSK3 at a serine near the $\mathrm{N}$ terminus to generate an autoinhibitory peptide that blocks substrate access. In contrast, several models suggest that Wnt signaling promotes GSK3 inhibition by translocating GSK3 to the membrane, where it directly interacts with the phosphorylated co-receptor LRP5/6. To examine the effects of GSK3 inhibition on protein conformation, Stamos et al. obtained the crystal structure of GSK3 bound to the $\mathrm{N}$-terminal phosphorylated peptide or phosphorylated LRP6 motifs. The authors found that these inhibitory peptides occupied the substrate binding pocket, acting as pseudo-substrates that promote conformational changes in the ' $\mathrm{C}$-loop' and the glycine-rich N-terminal loop, indicative of a catalytically active structure. The C-loop rearrangement resulted in direct interactions with the inhibitory peptides. Finally, the authors identified residues on both GSK3 (Phe93) and on the inhibitor peptide (positions 2 and 5 after the phosphorylation site) that mediate substrate binding. Overall, these structural observations provide useful insight that will inform future development of specific GSK3 inhibitors.

$G M$

Written by Mirella Bucci, Joshua M. Finkelstein,

Catherine Goodman, Grant Miura and

Terry L. Sheppard 